Check for updates

Cite this: Phys. Chem. Chem. Phys., 2018, 20, 7549

Received 7th February 2018, Accepted 12th February 2018

DOI: $10.1039 / c 8 c p 00884 a$

rsc.li/pccp

\section{Singlet and triplet energy transfer dynamics in self-assembled axial porphyrin-anthracene complexes: towards supra-molecular structures for photon upconversion $\dagger$}

\author{
Victor Gray, (D) Betül Küçüköz, (D) Fredrik Edhborg, Maria Abrahamsson, \\ Kasper Moth-Poulsen (D) and Bo Albinsson (D)*
}

\begin{abstract}
Energy and electron transfer reactions are central to many different processes and research fields, from photosynthesis and solar energy harvesting to biological and medical applications. Herein we report a comprehensive study of the singlet and triplet energy transfer dynamics in porphyrin-anthracene coordination complexes. Seven newly synthesized pyridine functionalized anthracene ligands, five with various bridge lengths and two dendrimer structures containing three and seven anthracene units, were prepared. We found that triplet energy transfer from ruthenium octaethylporphyrin to an axially coordinated anthracene is possible, and is in some cases followed by back triplet energy transfer to the porphyrin. The triplet energy transfer follows an exponential distance dependence with an attenuation factor, $\beta$, of $0.64 \AA^{-1}$. Further, singlet energy transfer from anthracene to the ruthenium porphyrin appears to follow a $R^{6}$ Förster distance dependence. Porphyrin-anthracene complexes are also used as triplet sensitizers for triplet-triplet annihilation (TTA) based photon upconversion, demonstrating their potential for photophysical and photochemical applications. The triplet lifetime of the complex is extended by the anthracene ligands, resulting in a threefold increase in the upconversion efficiency, $\Phi_{\cup c}$ to $4.5 \%$, compared to the corresponding ruthenium porphyrin-pyridine complex. Based on the results herein we discuss the future design of supra-molecular structures for TTA upconversion.
\end{abstract}

Porphyrins, the heterocyclic $18 \pi$-electron conjugated system common in many natural and biological systems, and their related structures are widely studied. In nature, they are essential for light harvesting, energy and electron transfer reactions, catalysis and oxygen transport. ${ }^{1-3}$ Accordingly, scientists have studied porphyrin compounds and developed numerous model systems to understand and exploit these versatile and useful compounds. A variety of uses have been explored, such as: light harvesting antennas for artificial photosynthesis and solar energy conversion devices; ${ }^{4-12}$ singlet oxygen sensitizers for photodynamic therapy; ${ }^{13}$ wires in molecular electronics; ${ }^{14-17}$ and triplet sensitizers for triplet-triplet annihilation based photon upconversion, ${ }^{18-21}$ to name a few. In both the natural and man-made systems, understanding the mechanisms of energy and electron transfer reactions is crucial. For example, in plants and photosynthetic bacteria triplet energy transfer (TET) occurs

Chalmers University of Technology, Department of Chemistry and Chemical Engineering, Gothenburg, Sweden. E-mail: balb@chalmers.se

$\dagger$ Electronic supplementary information (ESI) available: Synthetic procedures and NMR spectra of the new ligands, description of the fitting procedures and the corresponding photophysical data, time resolved and steady state phosphorescence data, transient absorption spectra. See DOI: 10.1039/c8cp00884a from the light-absorbing chlorophyll moiety to pendant carotenoids as a way of protecting the biological species against reactive singlet oxygen. ${ }^{22-24}$ Engineered photosynthetic systems with increased efficiencies will require even greater oxygen protection, motivating further exploration of TET in model porphyrin donor-acceptor systems. ${ }^{24}$ Furthermore, in the development of supramolecular structures designed for photochemical applications, such as triplet-triplet annihilation based photon upconversion $^{25-27}$ or photocatalysis ${ }^{28-30}$ the delicate balance between energy and electron transfer reactions requires careful tuning.

As part of the ongoing studies of energy and electron transfer reactions in porphyrin donor-acceptor system for various photochemical and electrochemical applications, ${ }^{4,5,7,24,25,31-44}$ we present a detailed photophysical study of a series of new, well-defined, self-assembled, axially coordinated ruthenium porphyrin-anthracene donor-acceptor systems. These systems consist of $2,3,7,8,12,13,17,18$-octaethylporpyrin ruthenium(II) carbonyl (RuOEP(CO)) coordinated axially by five different pyridine functionalized anthracene based ligands with various bridge lengths (8-25 $\mathrm{A}$ ) or two different dendrimer ligands with 3-7 monomer units. TET from the porphyrin to the anthracene 
ligand and singlet energy transfer (SET) in the opposite direction are consequently studied as a function of bridge length, and ligand dendrimer size. Transient absorption spectroscopy and time resolved phosphorescence spectroscopy are combined with steady state techniques to study the energy landscape of the complexes. We find that TET to the ligand extends the triplet lifetime of the complex from $20 \mu \mathrm{s}$ up to $600 \mu \mathrm{s}$. With an extended triplet lifetime the sensitization capabilities are improved and we employ the complexes as sensitizers for sensitized triplet-triplet annihilation photon upconversion (TTA-UC). Aiming towards efficient TTA-UC materials in the solid state we further discuss the capabilities of these complexes as sensitizers in supra-molecular TTA-UC systems. As such, this comprehensive study of anthracene-porphyrin complexes unravels important design principles for supra-molecular systems achieving efficient energy transfer.

\section{Results and discussion}

\subsection{Ligand design and synthesis}

Ligand design is based on our previous work with 9,10-diphenylanthracene (DPA) based ligands and dendrimeric structures. ${ }^{25,45}$ Fig. 1 shows the structures of the ligands: ligand 1 has a metapyridine substitution, ligands 2-5 have para-pyridine substitutions with increasing bridge lengths and dendrimeric ligands 6 and 7 have an increasing number of DPA monomer units. Fig. 1 also shows the structure of the triplet sensitizer octaethylporphyrin ruthenium(II) carbonyl (RuOEP(CO)).

The synthesis of ligands 1-5 was reported previously. ${ }^{25}$ The dendrimer ligands were synthesized by repeating a sequence of three high yielding and simple reactions; bromination followed

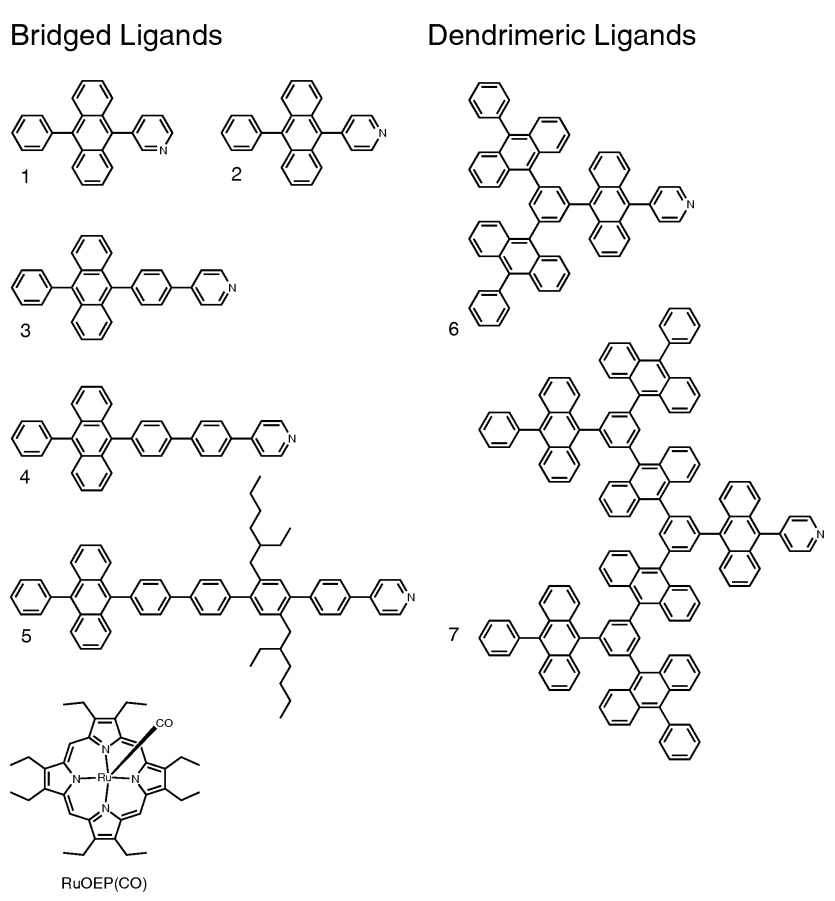

Fig. 1 Structures of the studied anthracene ligands 1-7 and the octaethylporphyrin ruthenium(॥) carbonyl RuOEP(CO). by borylation followed by a Suzuki-coupling, similar to our previously established route for DPA dendrons and dendrimers. ${ }^{45}$ The synthetic route is illustrated in Fig. S1 and described in more detail in the ESI. $\dagger$ Similar to what was reported previously RuOEP(CO) was obtained by refluxing the corresponding freebase porphyrin with $\mathrm{Ru}_{3} \mathrm{CO}_{12} \cdot{ }^{46-48} \ddagger$

\subsection{Binding equilibrium}

Upon mixing the porphyrin RuOEP(CO) with pyridine (Pyr) or the pyridine containing ligands 1-7, pyridine-porphyrin complexes RuOEP(CO)L ( $\mathbf{L}=\mathbf{1 - 7}$ or Pyr) are formed. The binding is followed using UV/Vis absorption spectroscopy, as seen in Fig. 2 and Fig. S8 (ESI $\dagger$ ). Upon binding to pyridine the porphyrin Q-band absorption is red-shifted ${ }^{49,50}$ and the complex Q-band has absorption peaks at 549 and $518 \mathrm{~nm}$.

The pyridine-ruthenium porphyrin bond is very strong, with a binding constant in the order of $10^{6}-10^{7} \mathrm{M}^{-1}$ (ESI, $\dagger$ Table S1), to be compared to the pyridine-zinc porphyrin bond of $6000 \mathrm{M}^{-1}$, reported in our previous work. ${ }^{25}$ The larger binding constant leads to about $90 \%$ of the ligand being bound to the porphyrin at a $1: 1$ mixture of RuOEP(CO) (total porphyrin concentration is $0.1 \mathrm{mM}$ ) and $\mathbf{L}$ (total ligand concentration is $0.1 \mathrm{mM}$ ). To form significant amounts of the zinc porphyrin-pyridine ligand complexes large excess of the ligand is required. The ruthenium based complexes are therefore better suited for fundamental studies since welldefined complexes can easily be formed.

\subsection{Triplet energy transfer and phosphorescence quenching}

RuOEP(CO)Pyr is slightly phosphorescent in degassed toluene solutions, Fig. S9 (ESI $\dagger$ ), with a quantum yield $\left(\Phi_{\mathrm{P}}\right)$ of $0.68 \%$. From the phosphorescence onset the triplet state energy ${ }^{3} \mathbf{R u O E P}(\mathbf{C O}) \mathbf{P y r}{ }^{*}$ is estimated to be $1.90 \mathrm{eV}(650 \mathrm{~nm})$, corresponding to a singlettriplet excited state splitting of $0.35 \mathrm{eV}$.

The triplet energy of the anthracene ligands is expected to be similar to that of DPA, $1.77 \mathrm{eV} .^{51,52}$ Therefore, a small driving force for TET from the porphyrin to the anthracene ligand exists. A common way of studying TET from phosphorescent porphyrins is by monitoring the phosphorescence quenching of the sensitizer by an acceptor. As can be seen in Table 1 there is only partial quenching of the RuOEP(CO)L phosphorescence compared to RuOEP(CO)Pyr. At first this low degree of quenching and that all compounds show similar phosphorescence quantum yields might seem surprising; its origin will be discussed in the following paragraph.

Nanosecond resolved transient absorption (TA) and time resolved phosphorescence measurements were both performed to further study the TET process, and the results are summarized in Table 2. The TA spectra of the RuOEP(CO)L complexes are found in Fig. 3 and Fig. S10 (ESI $\dagger$ ). RuOEP(CO)Pyr displays strong $\mathrm{T}_{1}-\mathrm{T}_{n}$ excited state absorption (ESA) in the $400-500 \mathrm{~nm}$ region and a distinct ground state bleach (GSB) at $520 \mathrm{~nm}$ and $550 \mathrm{~nm}$, in accordance with the ground state absorption spectra. Both the ESA and GSB decay with a time constant of $20 \mu \mathrm{s}$, in accordance with the phosphorescence decay, vide infra.

$\ddagger$ RuOEP(CO) is a low-spin d-6 complex, with a singlet ground-state. ${ }^{47}$ 

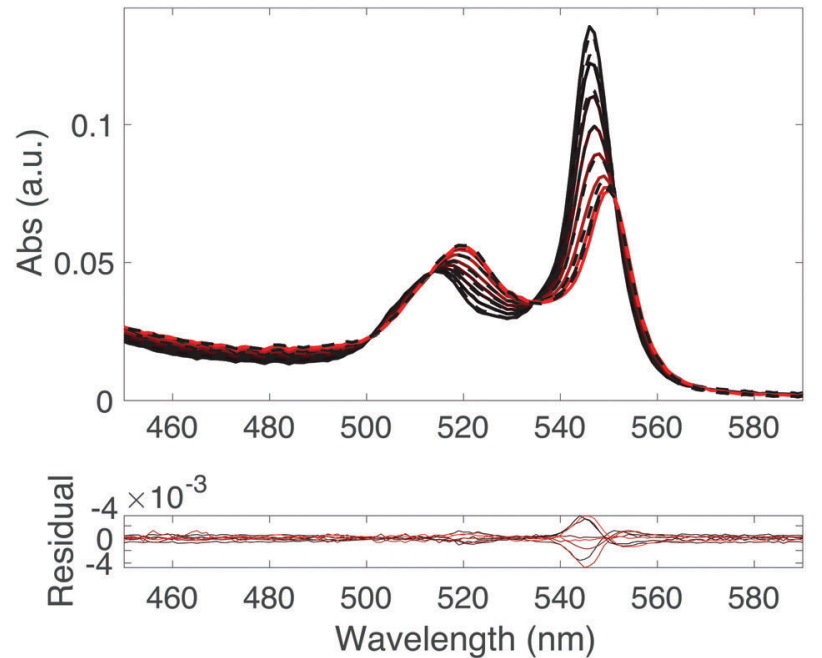

Fig. 2 Shift in RuOEP(CO) $(0.1 \mathrm{mM})$ absorption as ligand 1 is added $(0-0.1 \mathrm{mM})$ to form complex RuOEP(CO)1. Measured (dashed) and predicted (solid) spectra with residual below. Titration progression from black to red spectra.

Table 1 Phosphorescence quantum yield $\left(\Phi_{\mathrm{p}}\right)$ of RuOEP(CO)L complexes with pyridine (Pyr) and ligands 1-7; triplet energy transfer rates between the ruthenium porphyrin and the ligand; and triplet lifetimes of the ligand $\left(\tau_{\mathrm{TL}}\right)^{\mathrm{a}}$

\begin{tabular}{lllll}
\hline Ligand & $\Phi_{\mathrm{p}}(\%)$ & $k_{\text {TET }}\left(\mathrm{s}^{-1}\right)$ & $k_{\text {bTET }}\left(\mathrm{s}^{-1}\right)$ & $\tau_{\mathrm{TL}}(\mu \mathrm{s})$ \\
\hline Pyr & $0.68 \pm 0.10$ & - & - & - \\
$\mathbf{1}$ & $0.29 \pm 0.07$ & $>10 \times 10^{9}$ & $>2 \times 10^{8}$ & 680 \\
$\mathbf{2}$ & $0.29 \pm 0.01$ & $3.6 \times 10^{7}$ & $7.3 \times 10^{5}$ & 735 \\
$\mathbf{3}$ & $0.29 \pm 0.01$ & $7.6 \times 10^{5}$ & $2.1 \times 10^{4}$ & 500 \\
$\mathbf{4}$ & $0.30 \pm 0.05$ & $8.4 \times 10^{4}$ & $1.7 \times 10^{3}$ & 190 \\
$\mathbf{5}$ & $0.42 \pm 0.12$ & $3.3 \times 10^{4}$ & $0.2 \times 10^{3}$ & 315 \\
$\mathbf{6}$ & $0.19 \pm 0.01$ & $3.7 \times 10^{7}$ & $3.4 \times 10^{5}$ & 820 \\
$\mathbf{7}$ & $0.11 \pm 0.01$ & $3.8 \times 10^{7}$ & $2.4 \times 10^{5}$ & 610
\end{tabular}

${ }^{a}$ Phosphorescence quantum yields, $\Phi_{\mathrm{P}}$ determined in degassed toluene relative to Cresyl violet in methanol. $k_{\mathrm{TET}}$ is the triplet energy transfer (TET) rate, $k_{\mathrm{bTET}}$ is the rate of back TET and the intrinsic lifetime of the ligand is $\tau_{\mathrm{TL}}=k_{\mathrm{TL}}{ }^{-1}$.

Table 2 Decay times of RuOEP(CO)L in degassed toluene determined from transient absorption $(T A)^{a}$ and phosphorescence $(p)^{b}$ decays

\begin{tabular}{lclll}
\hline Ligand & $\tau_{1}^{\mathrm{TA}}(\mu \mathrm{s})$ & $\tau_{1}^{\mathrm{P}}(\mu \mathrm{s})$ & $\tau_{2}^{\mathrm{TA}}(\mu \mathrm{s})$ & $\tau_{2}^{\mathrm{P}}(\mu \mathrm{s})$ \\
\hline $\mathbf{P y r}$ & 20 & 17 & - & - \\
$\mathbf{1}$ & $<0.0001$ & - & 400 & 320 \\
$\mathbf{2}$ & 0.027 & - & 430 & 510 \\
$\mathbf{3}$ & 1 & - & 310 & 290 \\
$\mathbf{4}$ & 7 & 7 & 170 & 240 \\
$\mathbf{5}$ & 12 & 15 & 300 & - \\
$\mathbf{6}$ & 0.027 & - & 600 & 270 \\
7 & 0.027 & - & 520 & 190
\end{tabular}

${ }^{a}$ Excited at $550 \mathrm{~nm}$ and monitored at $440 \mathrm{~nm}$ and $520 \mathrm{~nm} .{ }^{b}$ Excited at $405 \mathrm{~nm}$ and emission monitored at $650 \mathrm{~nm}$.

With the pyridine anthracene ligand 2 the ground state bleach decays with $\tau=27 \mathrm{~ns}$, Table 2 , as does the strong porphyrin $\mathrm{T}_{1}-\mathrm{T}_{n}$ ESA, which decays into a longer lived ( $\tau=430 \mu \mathrm{s})$, weaker ESA in the same $400-500 \mathrm{~nm}$ region. This weaker ESA feature corresponds to the $\mathrm{T}_{1}-\mathrm{T}_{n}$ transition of the anthracene unit. The fast decay of the porphyrin centered triplet followed by the observation of an excited anthracene triplet suggest that there is efficient TET from the porphyrin to the ligand; ${ }^{3} \operatorname{RuOEP}^{*}(\mathbf{C O}) \mathbf{L} \rightarrow$ $\operatorname{RuOEP}(\mathbf{C O})^{3} \mathbf{L}^{*}$. For anthracene ligands with longer bridges the ${ }^{3}$ RuOEP$^{*}(\mathbf{C O}) \mathbf{L}$ triplet decays slower, Fig. 3 and Fig. S10 (ESI $\dagger$ ), indicating a slower TET, as expected by the exponential distance dependence of TET. ${ }^{36,37,40}$

For the larger dendrimeric ligands, 6 and 7, the porphyrin triplet also decays in about $27 \mathrm{~ns}$, the same as for 2, Fig. S10 (ESI $\dagger$ ). As the shortest porphyrin-anthracene distance is expected to be the same in these three complexes, the TET rate is expected to be similar. The observed longer decay time increases slightly in the larger ligands, from $430 \mu \mathrm{s}$ to $600 \mu \mathrm{s}$ and $520 \mu \mathrm{s}$ for $\mathbf{2 , 6}$ and 7 , respectively. Ligand $\mathbf{1}$, with a meta-pyridine, displays the fastest decay of the porphyrin triplet, faster than the time-resolution of our TA setup. The expected tilted binding in $\operatorname{RuOEP}(\mathbf{C O}) \mathbf{1}$ most likely increases the orbital overlap between the porphyrin triplet, located on the ring $\pi$-system, and the anthracene unit, explaining the increased TET rate.

Since the TET is only slightly exergonic in the studied systems $(\sim 0.13 \mathrm{eV})$ it is also possible for TET back (bTET) from the anthracene ligand to the porphyrin to occur; $\operatorname{RuOEP}(\mathbf{C O})^{3} \mathbf{L}^{*} \rightarrow$ ${ }^{3}$ RuOEP $^{*}(\mathbf{C O}) \mathbf{L}$. In other words, the two states could be in equilibrium; ${ }^{3} \mathbf{R u O E P}^{*}(\mathbf{C O}) \mathbf{L} \rightleftharpoons \operatorname{RuOEP}(\mathbf{C O})^{3} \mathbf{L}^{*}$. To obtain the actual TET rate, both the forward and backward TET must be considered when analysing the biexponential decays obtained from the TA-measurements. Eqn (1) and (2) describe the relationship between the observed decay times $\tau_{1}$ and $\tau_{2}$ (Table 2) with the rate of TET and bTET, respectively:

$$
\begin{gathered}
k_{\mathrm{TET}}=\frac{k_{\mathrm{TP}}\left(\tau_{1}^{-1}+\tau_{2}^{-1}-k_{\mathrm{TP}}\right)-\tau_{1}^{-1} \tau_{2}^{-1}}{k_{\mathrm{TP}}-k_{\mathrm{TL}}}, \\
k_{\mathrm{bTET}}=\frac{-k_{\mathrm{TL}}\left(\tau_{1}^{-1}+\tau_{2}^{-1}-k_{\mathrm{TL}}\right)+\tau_{1}^{-1} \tau_{2}^{-1}}{k_{\mathrm{TP}}-k_{\mathrm{TL}}},
\end{gathered}
$$

where $k_{\mathrm{TP}}$ and $k_{\mathrm{TL}}$ are the intrinsic triplet decay rates of the porphyrin and ligand, respectively. Eqn (1) and (2) can be derived from the set of coupled rate equations governing the system. ${ }^{53}$ A modified expression for the phosphorescence quantum yield, $\Phi_{\mathrm{p}}$, is obtained considering TET and bTET, as described in the ESI. $\dagger$ Assuming an intersystem crossing yield of unity for $\mathbf{R u O E P}(\mathbf{C O}) \mathbf{L},{ }^{54}$ the phosphorescence quantum yield is given by eqn (3):

$$
\Phi_{\mathrm{p}}=\frac{k_{\mathrm{r}}}{k_{\mathrm{TP}}+k_{\mathrm{TET}}-k_{\mathrm{bTET}} \times \frac{k_{\mathrm{TET}}}{k_{\mathrm{TL}}+k_{\mathrm{bTET}}}}
$$

where $k_{\mathrm{TP}}$ and $k_{\mathrm{TL}}$ are the intrinsic triplet decays of the porphyrin and anthracene ligand, respectively. For the derivation of eqn (3) the reader is referred to the ESI. $\dagger$ Combining eqn (1)-(3) one can calculate $k_{\mathrm{TET}}, k_{\mathrm{bTET}}$ and $k_{\mathrm{TL}}$. The obtained rates for TET and bTET can be found in Table 1 together with the intrinsic triplet lifetimes of the ligands, $\tau_{\mathrm{TL}}={k_{\mathrm{TL}}}^{-1}$.

For the shortest bridges the effect of bTET is small and $k_{\mathrm{TET}}$ is similar to $\tau_{1}{ }^{-1}$. In complexes with larger donor-acceptor separation the TET and bTET are slower, and the effect of bTET 
a)

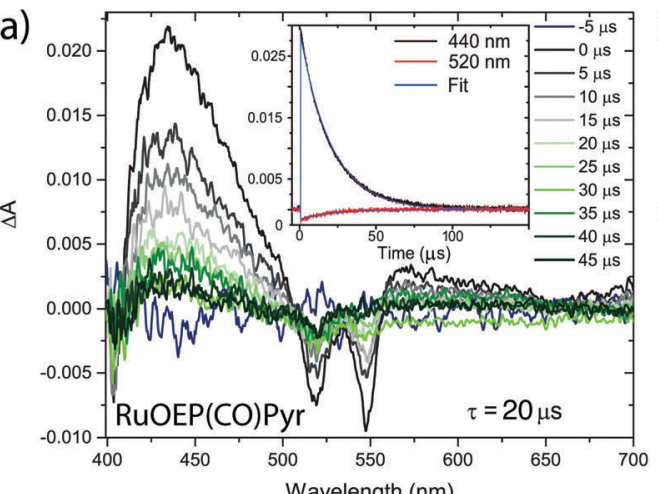

c)

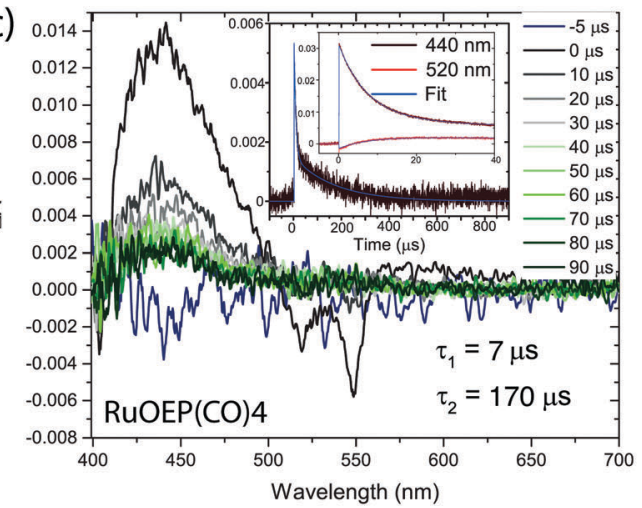

b)

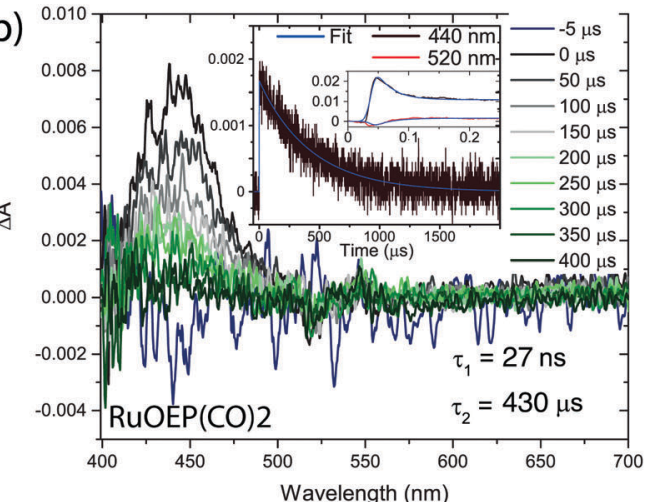

d)

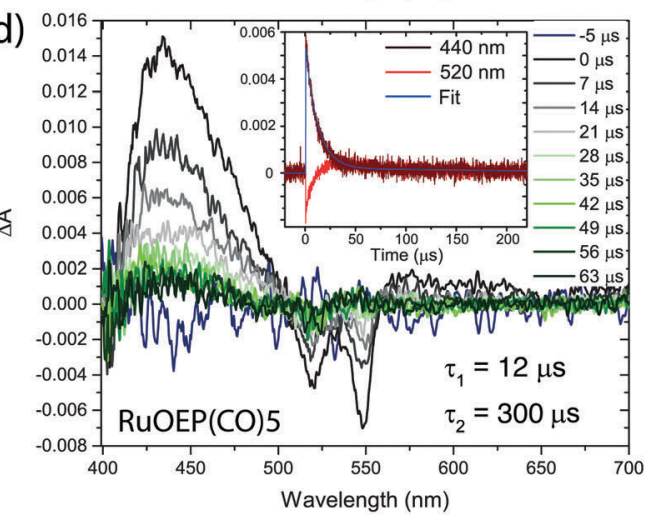

Fig. 3 Transient absorption spectra of (a) RuOEP(CO)Pyr, (b) RuOEP(CO)2, (c) RuOEP(CO)4 and (d) RuOEP(CO)5. All samples excited at $550 \mathrm{~nm}$ in degassed toluene.

is larger. Due to microscopic reversibility the rate $k_{\mathrm{bTET}}$ is related to $k_{\mathrm{TET}}$ through the Boltzmann factor as described in eqn (4):

$$
k_{\mathrm{bTET}}=k_{\mathrm{TET}} \exp \left(\frac{-\Delta E}{k_{\mathrm{b}} T}\right)
$$

where $k_{\mathrm{b}}$ is the Boltzmann constant, $T$ is the temperature and $\Delta E$ is the free energy difference between the triplet states of RuOEP(CO) and L, estimated spectroscopically to be $0.13 \mathrm{eV}$ (vide supra). From the obtained rates we can alternatively estimate the energy splitting to $0.11 \mathrm{eV}$, in good agreement with that estimated above.

Furthermore, the energy transfer dynamics were also monitored for some of the samples using time-resolved phosphorescence, Table 2 and Fig. S11 (ESI $\dagger$ ). In $\mu$ s emission measurements, the donor only complex, RuOEP(CO)Pyr, displays a phosphorescence decay of $17 \mu \mathrm{s}$, comparable to that observed for the triplet decay in the TA measurements, Table 2. In the donor-acceptor complexes where the TET is below the time-resolution of our phosphorescence measurements only a longer lifetime is observed, supporting that bTET occurs resulting in the observed delayed phosphorescence. The dendrimeric ligand complexes RuOEP(CO)6 and RuOEP(CO)7 also show mono exponential delayed phosphorescence lifetimes. In complexes RuOEP(CO)4 and RuOEP(CO)5, however, a biexponential decay is observed and in both cases the shorter of the two lifetimes are in good agreement to the TET rate determined from TA measurements. The longer lifetime is again longer than the donor only complex, further supporting bTET as an explanation to the minor phosphorescence quenching. The magnitude of the long lifetimes agrees well between experiments, there is however some discrepancy between the exact lifetimes in the different experiments. One reason can be that in nanosecond TA spectroscopy the long lived ligand state is directly probed and its decay is accurately monitored. In the phosphorescence measurements, on the other hand, the emissive porphyrin triplet state is monitored and the long lived ligand state is thus monitored indirectly. Therefore, the long lived component is expected to only make up a few percent of the total decay making it difficult to measure with the same accuracy.

Fig. 4 shows the dependence of the TET rate as a function of donor-acceptor distance, $R_{\mathrm{DA}}$. Since TET processes proceed through an electron exchange mechanism an exponential distance dependence is expected: ${ }^{36,37}$

$$
k_{\mathrm{TET}}=k_{0} \exp \left(-\beta R_{\mathrm{DA}}\right)
$$

where $k_{0}$ is the rate of TET at zero donor-acceptor separation and $\beta$ is an attenuation factor. Here $R_{\mathrm{DA}}$ is defined as the distance between the anthracene core and the porphyrin metal centre obtained from optimized structures of the RuOEP(CO)2RuOEP(CO)5 complexes.§ For the three complexes with the shortest separation (RuOEP(CO)L, L $=2-\mathbf{2}) k_{\mathrm{TET}}$ decreases

$\S$ Optimized structures of the RuOEP(CO)L coordination complexes were modelled with PM3 as implemented in the HyperChem 8.0 computational chemistry package. 


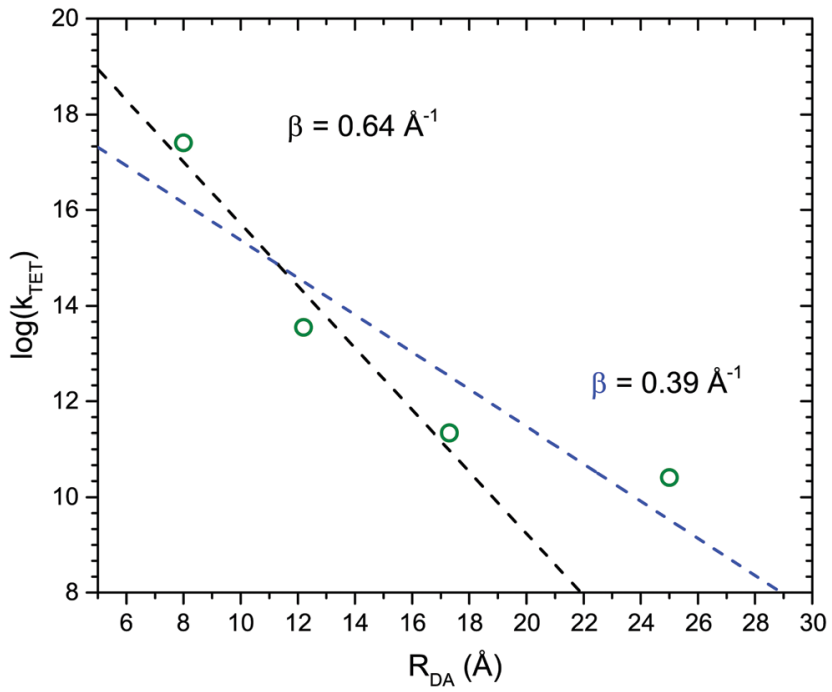

Fig. 4 Triplet energy transfer rates $\left(k_{\mathrm{TET}}\right)$ as a function of donor-acceptor separation $\left(R_{\mathrm{DA}}\right)$ in toluene. The triplet energy transfer rate decreases exponentially with $R_{\mathrm{DA}}$, with an attenuation factor $\beta=0.39$ or $0.64 \AA^{-1}$, depending on if the longest bridge (RuOEP(CO)5, $\left.R_{\mathrm{DA}}=25 \AA\right)$ is included in the linear fit or not.

exponentially with $R_{\mathrm{DA}}$ and fits nicely with an attenuation factor $\beta$ of $0.64 \AA^{-1}$. The TET in RuOEP(CO)5 does not follow this exponential dependence. A possible explanation of the deviation from exponential dependence is the change of the transfer mechanism, from a tunnelling to a hopping mechanism. ${ }^{55}$ However, the triplet energy of unsubstituted poly $p$-phenylene with 5 phenyl units is about $2.3 \mathrm{eV}$, substantially uphill from the RuOEP(CO) triplet state at $1.90 \mathrm{eV} \cdot{ }^{56}$ Possibly the disubstitution of 2,6-ethylhexyl on the central phenyl group, the terminal pyridine and the coordination to the ruthenium atom results in a slightly stabilized triplet energy of the bridge enabling hopping. It can, however, not be ruled out that RuOEP(CO)5 also follows an exponential distance dependence, in that case $\beta$ for the entire series is $0.39 \AA^{-1}$. Including $\operatorname{RuOEP}(\mathbf{C O}) 5$ in the analysis results in a worse linear fit, however, $0.39 \AA^{-1}$ and $0.64 \AA^{-1}$ both fall in the range of reasonable $\beta$ values for $p$-phenylene bridges. ${ }^{36,57-59}$

\subsection{Singlet energy transfer from anthracene ligands to RuOEP(CO)}

Due to the large singlet-triplet splitting in anthracene chromophores, 1.3-1.4 eV, the anthracene ligands function either as triplet acceptors or singlet donors in the studied RuOEP(CO)L complexes. Upon complex formation the prompt anthracene emission is quenched by more than $95 \%$, indicating efficient singlet energy transfer (SET) to the porphyrin. Fig. 5 displays the ps-resolved fluorescence decays of complexes with ligands 3-5 and 7; fluorescence decays of ligands with shorter bridges could not be resolved on the current setup. The decays are biexponential, where the fast component is attributed to SET and the longer component to residual emission from unbound ligand. The fraction of the long component in the decay is not directly related to the fraction of unbound species in

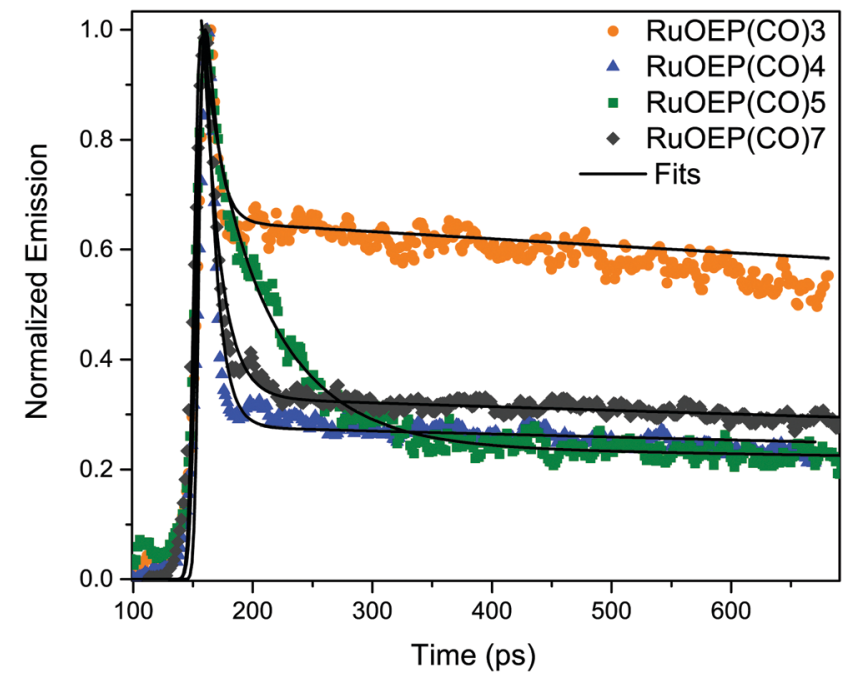

Fig. 5 Fluorescence decays of complexes RuOEP(CO)L, where $L$ is 3, 4, 5 or 7. Samples excited at $375 \mathrm{~nm}$ and decays are the average emission between 415-440 $\mathrm{nm}$.

the sample, as the lifetime of the free ligand is longer than the repetition rate $(80 \mathrm{MHz})$ a long lived emission signal is built up over time. However, the fact that complexes with ligands $\mathbf{4}$, 5 or 7 all have similar fractions of long lived components indicate that the amount of free ligand is similar in all samples, as expected. From the binding constants we estimate that $<10 \%$ free ligand is present in the samples. The much larger fraction of the long lived component of complex $\mathbf{R u O E P ( C O ) 3 ~ r e s u l t s ~ f r o m ~}$ the fact that the fast component is shorter than the pulse width. We can still obtain a range of reasonable values for the short component for RuOEP(CO)3 by expecting a similar fraction of the long lived component and deconvolving the signal with a reasonable pulse width. Further details of the fitting can be found in the ESI. $\dagger$

The SET in complex RuOEP(CO)5 occurs with $\tau_{\text {SET }}=48 \mathrm{ps,}$ Table 3 . As expected, shorter bridges exhibit faster SET, $\tau_{\mathrm{SET}}$ is about 5 ps for complex RuOEP(CO)4. Shortening the bridge further, as in complex RuOEP(CO)3, results in a SET that approaches the limit of our time resolution, observed as an increased fraction of the long lived component in the decay. As discussed above we can

Table 3 Ligand fluorescence lifetimes in degassed toluene ${ }^{a}$

\begin{tabular}{lll}
\hline Ligand & $\tau_{\text {SET }}(\mathrm{ps})$ & $\tau_{0}{ }^{b}(\mathrm{~ns})$ \\
\hline $\mathbf{1}$ & - & 7.1 \\
$\mathbf{2}$ & $-\left(0.1^{c}\right)$ & 7.1 \\
$\mathbf{3}$ & $0.5-3\left(0.8^{c}\right)$ & 4.3 \\
$\mathbf{4}$ & $5 \pm 1\left(5^{c}\right)$ & 3.8 \\
$\mathbf{5}$ & $48 \pm 2$ & 4.0 \\
$\mathbf{6}$ & - & 5.9 \\
7 & $8 \pm 1$ & 5.4
\end{tabular}

${ }^{a} \tau_{\mathrm{SET}}$ refers to the quenched lifetime of the anthracene fluorescence in the RuOEP(CO)L complex and $\tau_{0}$ is the unquenched lifetime. ${ }^{b}$ Determined for the ligand in separate TCSPC experiments and fixed in the analysis of the shorter lifetimes $\tau_{\mathrm{SET}}{ }^{c}{ }^{c}$ Lifetimes estimated assuming that the singlet energy transfer is solely governed by FRET, they should therefore be considered as an upper limit. 
still estimate the SET rate to be between 0.5 and 3 ps by assuming a similar fraction of residual fluorescence as observed in complexes RuOEP(CO)4, RuOEP(CO)5 and $\operatorname{RuOEP(CO)7.~In~complexes~}$ RuOEP(CO)1, RuOEP(CO)2 and RuOEP(CO)6 SET is much faster than our time resolution and only the long lived component is observed. It should be pointed out, however, that the steady state emission is quenched almost quantitatively in all complexes. Therefore, the long lived component must arise by residual amounts of unbound ligand, also in complexes RuOEP(CO)1, RuOEP(CO)2 and RuOEP(CO)6 where the decay component related to SET is not resolved. An interesting observation is that, in the complex with the largest dendrimeric ligand 7, SET is slowed down compared to the monomer ligand 2 and second generation dendrimer ligand $\mathbf{6}$. It indicates that on average the excited singlet state is located further away in the larger structures.

The rate of SET in complexes $\operatorname{RuOEP}(\mathbf{C O}) \mathbf{L}$, with $\mathbf{L}=\mathbf{3 - 5}$, follows a $R_{\mathrm{DA}}{ }^{6}$ dependence, Fig. S12 (ESI $\dagger$ ), indicating that SET is governed by FRET with a Förster distance $R_{0}=50 \AA$. Based on the FRET mechanism and taking the spectral overlap between ligand emission and porphyrin absorption into account, the lifetimes can be estimated to 0.1 ps for the shortest bridged ligand 2 in the complex, see the ESI $\dagger$ for details about the calculations. As can be seen in Table 3, the estimated lifetimes for complexes RuOEP(CO)3, 0.8 ps, and RuOEP(CO)4, 5 ps, are in good agreement with our experimental observations and also confirm that the expected lifetimes in the shortest bridged complex, $\operatorname{RuOEP}(\mathbf{C O}) 2$, are well below our time resolution.

$R_{0}$ for the RuOEP(CO)L complexes is about $10 \AA$ larger than for the corresponding zinc octaethylporphyrin (ZnOEPL) complexes, resulting in the SET in the current study being faster compared to similar complexes based on ZnOEP. ${ }^{25}$ For example, the SET in RuOEP(CO)5 is three times faster compared to the corresponding zinc complex ZnOEP5. The faster SET in the ruthenium complex cannot be explained by the difference in the Förster overlap integral $(J)$ as it actually is smaller for the case of the ruthenium complex. One significant difference between the zinc and ruthenium complexes is the ligand binding strength. The $\mathrm{Zn}-\mathrm{N}$ bond is quite weak $\left(K_{\text {bind }} \sim 10^{3}-10^{4} \mathrm{M}^{-1}\right)$, resulting in a dynamic ZnOEPL complex. With three orders of magnitude larger binding constant $\left(K_{\text {bind }} \sim 10^{6}-10^{7} \mathrm{M}^{-1}\right)$ the $\mathrm{Ru}-\mathrm{N}$ bond should be stronger, making the RuOEP(CO)L complex more stable. Therefore, one possible explanation for the faster SET in the ruthenium complexes could be that the through bond SET is greater in the stronger bonding ruthenium complex. ${ }^{37,60,61}$ With the longer ligands, however, where the porphyrin anthracene separation is large, close to $25 \AA$, it is expected that FRET is the dominating SET mechanism. Therefore, considering FRET, the faster SET could imply that the ligand can move away from the $90^{\circ}$ binding angle to a larger extent in the ruthenium case, as schematically described in Fig. 6. A greater deviation from the $90^{\circ}$ binding angle results in a more favourable geometry for FRET. For a detailed discussion on the angle dependence the reader is referred to ref. 25 . In short, an apparent binding angle can be estimated based on the fluorescence quenching experiments and a known spectral overlap. $^{25}$ For the $\mathbf{R u O E P}(\mathbf{C O}) \mathbf{L}$ complexes we estimate an

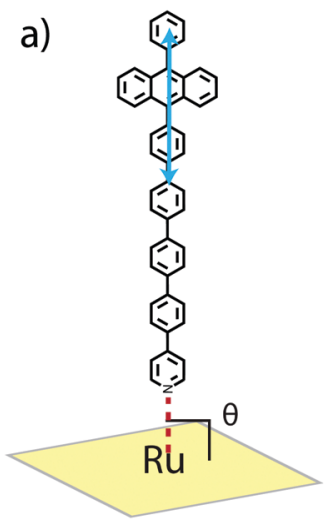

$\theta=90^{\circ}$, No FRET b)

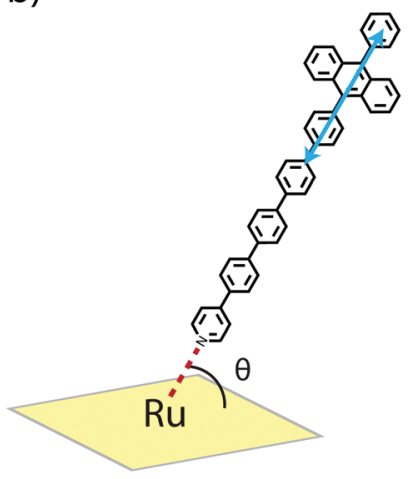

$\theta<90^{\circ}$, FRET possible
Fig. 6 Schematic illustration of an axially coordinated ruthenium-anthracene complex with (a) perpendicular binding and (b) non-perpendicular binding, relative to the porphyrin plane and anthracene transition dipole moment. In the studied systems movement is expected and only an apparent binding angle can be estimated from the FRET measurements.

apparent binding angle of $45^{\circ}$, compared to $>70^{\circ}$ in ZnOEPL. ${ }^{25}$ We find this large difference, however, highly unlikely, especially considering the stronger ruthenium-pyridine bond, possibly indicates that another process is also involved.

Based on the experimental results described above we can construct an energy level diagram for the RuOEP(CO)L complexes, as shown in Fig. 7. From the Marcus-Rehm-Weller equation, we estimate the energy of the charge separated state $\left(\mathbf{R u O E P}^{+}(\mathbf{C O}) \mathbf{L}^{-}\right)$to about $2.49 \mathrm{eV}$, for details of the calculation the reader is referred to the ESI. $\dagger$ The energy is estimated from redox potentials in acetonitrile and should therefore be considered

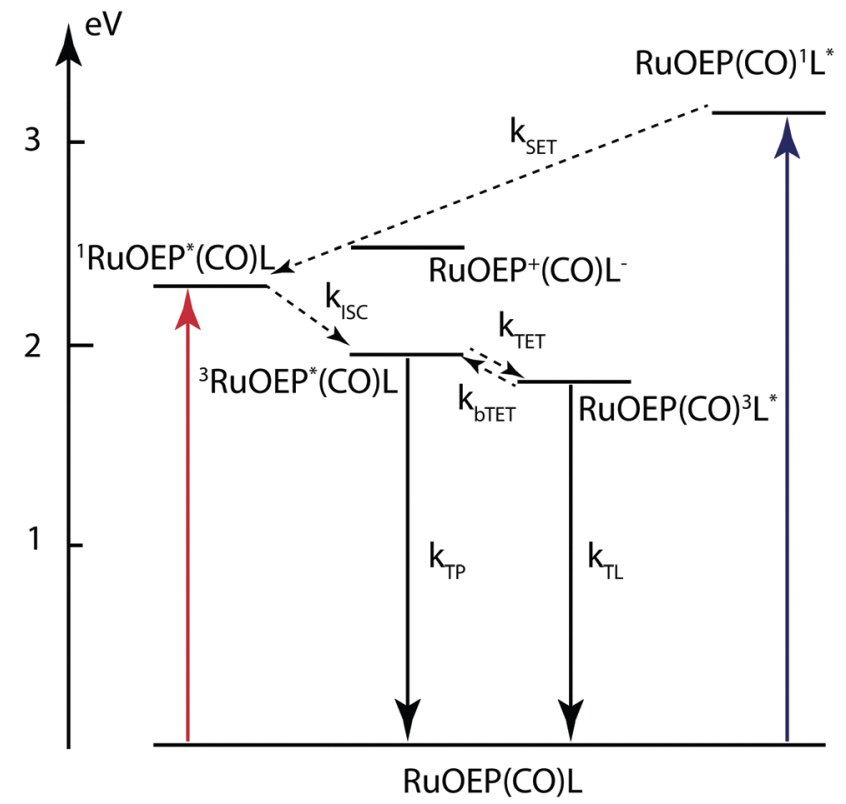

Fig. 7 Energy level diagram of RuOEP(CO)L complexes. The charge separated state $\mathbf{R u O E P}^{+}(\mathbf{C O}) \mathbf{L}^{-}$at $2.49 \mathrm{eV}$ is estimated from the redox potentials of the free components in acetonitrile and should therefore be considered a lower limit. 
as a lower limit since the current systems are studied in less polar toluene. As can be seen in Fig. 7 the energy of the charge separated state is accessible from the ligand singlet state $\operatorname{RuOEP}(\mathbf{C O})^{1} \mathbf{L}^{*}$. If populated, it would also contribute to the singlet quenching. Comparing the experimental quenching rates to a FRET governed distance dependence a reasonable $R^{6}$ dependence is followed as seen when comparing the slope 6 in the log-log plot in Fig. S12 (ESI $\dagger$ ) to the experimental values. However, the best linear fit is obtained for a slightly smaller slope of 5.4, possibly indicating that there is also another factor influencing the singlet quenching.

\subsection{RuOEP(CO)-ligand complexes applied to triplet-triplet annihilation photon upconversion}

Recently, many metallo-porphyrins have been used in sensitized triplet-triplet annihilation based photon upconversion (TTA-UC) systems. In TTA-UC systems the porphyrin triplet sensitizes an annihilator molecule through TET, the triplet excited annihilator can then undergo triplet-triplet annihilation (TTA) with another triplet excited annihilator forming a singlet excited annihilator which can emit a high energy photon. The most popular porphyrins for TTA-UC are based on palladium and platinum, ${ }^{20}$ probably due to their high intersystem crossing (ISC) yield, phosphorescent nature and stable structure. To the best of our knowledge, no ruthenium based porphyrins have been used as sensitizers for TTA-UC, even though they also have high ISC yields. $^{54}$

One of the main challenges faced in the field of TTA-UC is the development of solid state materials that maintain the high efficiencies observed in the liquid systems. The difficulty arises from the diffusion limited TET and TTA processes which are reduced in most solid systems. One approach is to develop large supra-molecular structures where TET and TTA can occur intra-molecularly, overcoming the diffusion limit. ${ }^{26}$ In such a supra-molecular system the sensitizer must be in close proximity to an annihilator. One advantage of ruthenium porphyrins, compared to the palladium and platinum analogues, is that pyridine ligands can coordinate to the ruthenium atom. By designing ligands that can accept the triplet energy, as described herein, the triplet energy transfer can be enhanced. We therefore used the RuOEP(CO)L complexes as sensitizers for TTA-UC with free DPA as an annihilator in toluene. It should be pointed out that the $\mathbf{R u O E P}(\mathbf{C O}) \mathbf{L}$ complexes are specifically designed to study the TET, thus TTA still must occur between two free annihilators, sensitized by two distinct complexes for upconversion to occur. As such the studied systems are only one part of a potential supra-molecular structure also incorporating intramolecular TTA. ${ }^{62}$

As can be seen in Fig. 8, when $\operatorname{RuOEP}(\mathbf{C O}) 2$ is used as a sensitizer, the upconversion quantum yield, $\Phi_{\mathrm{UC}}$, is increased about 3 times to about $4.5 \%$, $\uparrow$ compared to RuOEP(CO)Pyr which has $\Phi_{\mathrm{UC}}=1.5 \%$. The quantum yields are determined in

T All reported upconversion quantum yields are on the basis of a maximum of $50 \%$, two absorbed low energy photons can at most produce one high energy photon.

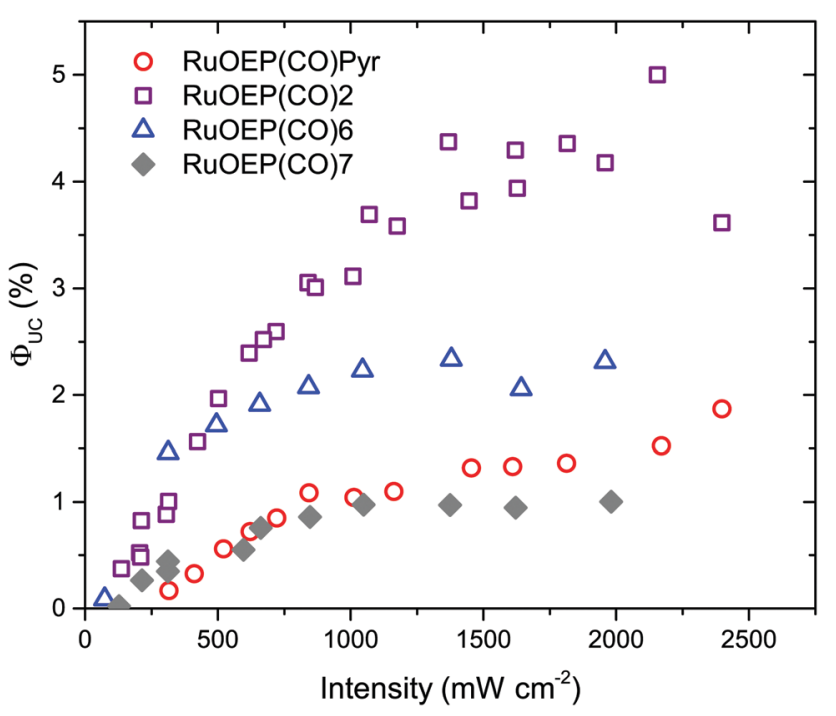

Fig. 8 Photon upconversion quantum yield, $\Phi_{\cup C}$, as a function of excitation intensity. Purple squares represent samples containing RuOEP(CO)2, blue triangles RuOEP(CO)6, grey diamonds RuOEP(CO)7 and red circles RuOEP(CO)Pyr as the sensitizer complex (19 $\mu \mathrm{M}$, porphyrin/ligand 1:1), 9,10-diphenylanthracene (DPA, $0.1 \mathrm{mM}$ ) is added as the annihilator. All samples in toluene, prepared in a glovebox $\left(<0.1 \mathrm{ppm} \mathrm{O}_{2}\right.$ and excited at $532 \mathrm{~nm})$.

the strong annihilation limit (linear regime), Fig. S13 (ESI $\dagger$ ). The increase in $\Phi_{\mathrm{UC}}$ can be understood in terms of the extended triplet lifetime of the $\operatorname{RuOEP}(\mathbf{C O}) 2$ complex. Fig. S14 and the corresponding section in the ESI $\dagger$ describe the effect on the TET efficiency and $\Phi_{\mathrm{UC}}$ by extending the triplet lifetime of the sensitizer. With the current annihilator concentration it is expected that RuOEP(CO)2 is approximately 1.5-2 times more efficient due to the increased TET efficiency, in line with our experimental observations.

Interestingly the effect of the extended triplet lifetime is not observed to the same extent for the complexes with dendrimeric ligands, RuOEP(CO)6 $\left(\Phi_{\mathrm{UC}}=2.3 \%\right)$ and RuOEP(CO)7 $\left(\Phi_{\mathrm{UC}}=1.0 \%\right)$, Fig. 8. $\Phi_{\mathrm{UC}}$ decreases more for the larger dendrimers. One possible explanation, in line with the observed decrease in $\Phi_{\mathrm{UC}}$, is a steric effect on the probability of free DPA colliding with the triplet excited monomer in the dendrimer ligand. This is a result of the current upconversion system still relying on diffusional TET and TTA. In fully supra-molecular structures where an annihilator dendrimer is coordinated to multiple sensitizers, allowing for both TTA and TET to occur intra-molecularly we envision that such problems can be circumvented.

\section{Conclusion}

Herein we report a comprehensive study of the energy transfer processes of seven new ruthenium porphyrin-anthracene coordination complexes, RuOEP(CO)L. The design and synthesis of pyridine containing anthracene ligands with various bridge lengths (8-25 $)$ ), consisting of 1-7 anthracene units, is described. The anthracene ligands bind strongly to the ruthenium atom 
resulting in close to quantitative binding for a $1: 1$ mixing ratio. Upon excitation of RuOEP(CO) the formed triplet is quickly transferred to the axially coordinated anthracene derivative. By separating the anthracene and porphyrin with phenyl bridges we observe a decrease in the triplet energy transfer rate, and an attenuation factor $\beta$ of $0.64 \AA^{-1}$ is determined. We further study the singlet energy transfer from the anthracene ligand to RuOEP(CO) using ps-resolved fluorescence lifetime measurements. The singlet anthracene is readily quenched by the porphyrin. By employing large dendrimeric structures or longer phenyl bridge separators the quenching is reduced. The singlet quenching follows a Förster type $R^{6}$ distance dependence, even though the transition dipole moments are close to perpendicularly oriented.

The current study is part of our broader investigation of supramolecular structures for TTA based photon upconversion. Here we demonstrate that by effectively extending the triplet lifetime of the ruthenium complex by coordination of the anthracene ligands, the upconversion quantum yield is increased threefold to $4.5 \%$ with complex RuOEP(CO)2. We have previously studied the properties of DPA based dendrimers and oligomers ${ }^{45,62}$ and shown that intra-molecular TTA is possible in such structures. Combined with the current study, where efficient intra-molecular TET is achieved, we are en route to developing fully functioning, well defined, supramolecular TTA upconversion systems, with broad implications spanning solar energy harvesting devices to bioimaging and drug targeting.

\section{Experimental}

Synthetic procedures for the new ligands 1, 6 and 7, and NMR spectra are found in the ESI. $\dagger$

\subsection{Photophysical characterization}

Steady-state absorption was recorded on a Cary 5000 spectrophotometer and steady state emission measurements were carried out on a Spex Flurolog 3 spectrofluorimeter (JY Horiba). Phosphorescence decays were carried out on the mentioned spectrofluorimeter. Unquenched fluorescence lifetimes were determined on a time correlated single photon counting (TCSPC) setup using a $377 \mathrm{~nm}$ laser diode (PicoQuant) and a MCP-PMT detector (10000 counts in the top channel, 2048 channels). Quenched fluorescence lifetimes were determined by time resolved emission measurements which were taken with a streak camera system; excitation pulses were generated using a Tsunami Ti:sapphire laser (Spectra-Physics) that was pumped by a Millennia Pro X laser (Spectra-Physics). The Tsunami laser output was set to $740 \mathrm{~nm}$ and frequency doubled to $370 \mathrm{~nm}$ using a frequency doubler/tripler (GWU, Spectra Physics) to excite the anthracene ligands. The emitted photons were passed through a spectrometer (Acton SP2300, Princeton Instruments) and were detected using a streak camera (C5680, Hamamatsu) with a synchroscan sweep unit (M5675, Hamamatsu).

Nanosecond transient absorptions measurements were performed on a home built system with a Surelite Continuum
Nd:YAG laser equipped with an Surelite Continuum OPO generating a 7 ns pump beam. A quartz-halogen lamp with a monochromator was used for the probe light. Either a CCD camera (iStar, Andor Technology) or a monochromator (Oriel Cornerstone 130, Newport) together with a 5 stage PMT (Applied Photophysics) coupled to an oscilloscope (TDS 2022, Tektronix) was used for recording the transient spectra or decay signal, respectively.

Upconversion samples were excited at $532 \mathrm{~nm}$ using a frequency doubled cw Nd:YAG laser (Millenia V, Spectra-Physics), the excitation intensity was varied using a neutral density filter and the intensity was measured using a power-meter from Starlite Ophir. The laser spot diameter was determined to be $2.5 \mathrm{~mm}$ using a laser alignment paper. The emission was recorded at a $90^{\circ}$ angle by out-coupling the emission with an optical fibre to an AvaSpec 2048 (Avantes) USB fibre spectrometer with a $532 \mathrm{~nm}$ notch filter between the sample and the fibre to protect the spectrometer from the intense scattered excitation light.

All photophysical measurements were carried out in toluene using quartz cuvettes. Samples were prepared in a glovebox from Innovative Technologies $\left(<0.1 \mathrm{ppm} \mathrm{O}_{2}\right)$ under a nitrogen atmosphere. RuOEP(CO)L phospohorescence quantum yields were determined using relative Cresyl violet in methanol $(\Phi=54 \%)$. Upconversion quantum yields, $\Phi_{\mathrm{UC}}$, were also determined using relative Cresyl violet in methanol employing the standard equation for relative fluorescence quantum yield determination:

$$
\Phi_{\mathrm{UC}}=\Phi_{\mathrm{r}} \frac{\left(1-10^{-A_{\mathrm{r}}}\right)}{\left(1-10^{-A_{\mathrm{x}}}\right)} \frac{F_{\mathrm{x}}}{F_{\mathrm{r}}} \frac{I_{\mathrm{r}}}{I_{\mathrm{x}}} \frac{\eta_{\mathrm{x}}^{2}}{\eta_{\mathrm{r}}^{2}}
$$

where $A$ is the absorbance, $F$ the corrected integrated emission, $I$ is the excitation intensity and $\eta$ is the refractive index of the medium. Subscripts $x$ and $r$ denote the sample and reference respectively.

\section{Conflicts of interest}

There are no conflicts of interest to declare.

\section{Acknowledgements}

The authors acknowledge funding from the Swedish Energy Agency, the Swedish Research Council, the Swedish Strategic Research Foundation and Knut \& Alice Wallenberg Foundation. Dr Martyn Jevric is acknowledged for providing compound 2.

\section{References}

1 M. F. Perutz, H. Muirhead, J. M. Cox and L. C. G. Goaman, Nature, 1968, 219, 131-139.

2 G. McDermott, S. M. Prince, A. A. Freer, A. M. HawthonthwaiteLawless, M. Z. Papiz, R. J. Cogdell and N. W. Isaacs, Nature, 1995, 374, 517-521.

3 T. Mirkovic, E. E. Ostroumov, J. M. Anna, R. van Grondelle and G. D. Scholes, Chem. Rev., 2017, 117, 249-293. 
4 M. R. Wasielewski, Chem. Rev., 1992, 92, 435-461.

5 D. Gust, T. A. Moore and A. L. Moore, Acc. Chem. Res., 1993, 26, 198-205.

6 D. Gust, T. A. Moore and A. L. Moore, Acc. Chem. Res., 2001, 34, 40-48.

7 G. Kodis, P. A. Liddell, L. D. Garza, P. C. Clausen, J. S. Lindsey, A. L. Moore, T. A. Moore and D. Gust, J. Phys. Chem. A, 2002, 106, 2036-2048.

8 Y. Nakamura, I. W. Hwang, N. Aratani, T. K. Ahn, D. M. Ko, A. Takagi, T. Kawai, T. Matsumoto, D. Kim and A. Osuka, J. Am. Chem. Soc., 2005, 127, 236-246.

9 D. Gust, T. A. Moore and A. L. Moore, Acc. Chem. Res., 2009, 42, 1890-1898.

10 K. Gao, L. Li, T. Lai, L. Xiao, Y. Huang, F. Huang, J. Peng, Y. Cao, F. Liu, T. P. Russel, R. A. J. Janssen and X. Peng, J. Am. Chem. Soc., 2015, 137, 7282-7285.

11 S. Mathew, N. A. Astani, B. F. E. Curchod, J. H. Delcamp, M. Marszalek, J. Frey, U. Rothlisberger, M. K. Nazeeruddin and M. Grätzel, J. Mater. Chem. A, 2016, 4, 2332-2339.

12 P. Qin, P. Sanghyun, M. I. Dar, K. Rakstys, H. Elbatal, S. A. Al-muhtaseb, C. Ludwig and M. K. Nazeeruddin, Adv. Funct. Mater., 2016, 26, 5550-5559.

13 J. Schmitt, V. Heitz, A. Sour, F. Bolze, H. Ftouni, J.-F. Nicoud, L. Flamigni and B. Ventura, Angew. Chem., Int. Ed., 2015, 54, 169-173.

14 G. Sedghi, K. Sawada, L. J. Esdaile, M. Hoffmann, H. L. Anderson, D. Bethell, W. Haiss, S. J. Higgins and R. J. Nichols, J. Am. Chem. Soc., 2008, 120, 8582-8583.

15 G. Sedghi, V. M. García-Suárez, L. J. Esdaile, H. L. Anderson, C. J. Lambert, S. Martín, D. Bethell, S. J. Higgins, M. Elliot, N. Bennett, J. E. Macdonald and R. J. Nichols, Nat. Nanotechnol., 2011, 6, 517-523.

16 Z. Li, T.-H. Park, J. Rawson, M. J. Therien and E. Borguet, Nano Lett., 2012, 12, 2722-2727.

17 G. Sedghi, L. J. Esdaile, H. Anderson, S. Martin, D. Bethell, S. J. Higgins and R. J. Nichols, Adv. Mater., 2012, 24, 653-657.

18 T. N. Singh-Rachford and F. N. Castellano, Coord. Chem. Rev., 2010, 254, 2560-2573.

19 J. Zhao, S. Ji and H. Guo, RSC Adv., 2011, 1, 937-950.

20 V. Gray, D. Dzebo, M. Abrahamsson, B. Albinsson and K. Moth-Poulsen, Phys. Chem. Chem. Phys., 2014, 16, 10345-10352.

21 C. E. McCusker and F. N. Castellano, Top. Curr. Chem., 2016, 374, 19.

22 T. G. Monger, R. J. Cogdell and W. W. Parson, Biochim. Biophys. Acta, 1976, 449, 136-153.

23 A. Gall, R. Berera, M. T. A. Alexandre, A. A. Pascal, L. Bordes, M. M. Mendes-pinto, S. Andrianambinintsoa, K. V. Stoitchkova, A. Marin, L. Valkunas, P. Horton, J. T. M. Kennis, R. V. Grondelle, A. Ruban and B. Robert, Biophys. J., 2011, 101, 934-942.

24 J. Ho, E. Kish, D. D. Méndez-Hernández, K. WongCarter, S. Pillai, G. Kodis, J. Niklas, O. G. Poluektov, D. Gust, T. A. Moore, A. L. Moore, V. S. Batista and B. Robert, Proc. Natl. Acad. Sci. U. S. A., 2017, 114, E5513-E5521.
25 V. Gray, K. Börjesson, D. Dzebo, M. Abrahamsson, B. Albinsson and K. Moth-Poulsen, J. Phys. Chem. C, 2016, 120, 19018-19026.

26 C. Fan, W. Wu, J. J. Chruma, J. Zhao and C. Yang, J. Am. Chem. Soc., 2016, 138, 15405-15412.

27 N. Yanai and N. Kimizuka, Chem. Commun., 2016, 52, 5354-5370.

28 F. Li, Y. Jiang, B. Zhang, F. Huang, Y. Gao and L. Sun, Angew. Chem., Int. Ed., 2012, 51, 2417-2420.

29 J. Liu, L. Chen, H. Cui, J. Zhang, L. Zhang and C.-Y. Su, Chem. Soc. Rev., 2014, 43, 6011-6061.

30 V. Bandi, F. P. D. Souza, H. B. Gobeze and F. D'Souza, Chem. - Eur. J., 2015, 21, 2669-2679.

31 K. Kilså, J. Kajanus, S. Larsson, A. N. Macpherson, J. Mårtensson and B. Albinsson, Chem. - Eur. J., 2001, 7, 2122-2133.

32 D. Holten, D. F. Bocian and J. S. Lindsey, Acc. Chem. Res., 2002, 35, 57-69.

33 A. Prodi, C. Chiorboli, F. Scandola, E. Iengo, E. Alessio, R. Dobrawa and F. Würtner, J. Am. Chem. Soc., 2005, 127, 1454-1462.

34 D. M. Guldi, G. M. A. Rahman, V. Sgobba and C. Ehli, Chem. Soc. Rev., 2006, 35, 471-487.

35 Y. Nakamura, N. Aratani and A. Osuka, Chem. Soc. Rev., 2007, 36, 831-845.

36 B. Albinsson, M. P. Eng, K. Pettersson and M. U. Winters, Phys. Chem. Chem. Phys., 2007, 9, 5847-5864.

37 B. Albinsson and J. Mårtensson, J. Photochem. Photobiol., C, 2008, 9, 138-155.

38 T. K. Khan, M. Bröring, S. Mathur and M. Ravikanth, Coord. Chem. Rev., 2013, 257, 2348-2387.

39 J. G. Woller, J. K. Hannestad and B. Albinsson, J. Am. Chem. Soc., 2013, 135, 2759-2768.

40 M. Gilbert and B. Albinsson, Chem. Soc. Rev., 2015, 44, 845-862.

41 A. Antoniuk-Pablant, G. Kodis, A. L. Moore, T. A. Moore and D. Gust, J. Phys. Chem. B, 2016, 120, 6697.

42 H. Dekkiche, A. Buisson, A. Langlois, P.-L. Karsenti, L. Ruhlmann, P. D. Harvey and R. Ruppert, Inorg. Chem., 2016, 55, 10329-10336.

43 D. R. Martir, G. J. Hedley, D. B. Cordes, A. M. Z. Slawin, D. Escudero, D. Jacquemin, T. Kosikova, D. Philp, D. M. Dawson, S. E. Ashbrook, I. D. W. Samuel and E. Zysmancolman, Dalton Trans., 2016, 45, 17195-17205.

44 Y. Hu, M. B. Thomas, R. G. W. Jinadasa, H. Wang and F. D’Souza, Chem. - Eur. J., 2017, 23, 12805-12814.

45 K. Börjesson, M. Gilbert, D. Dzebo, B. Albinsson and K. Moth-Poulsen, RSC Adv., 2014, 4, 19846.

46 M. Tsutsui, D. Ostfeld and L. M. Hoffman, J. Am. Chem. Soc., 1971, 93, 1820-1823.

47 A. Antipas, J. W. Buchler, M. Gouterman and P. D. Smith, J. Am. Chem. Soc., 1978, 100, 3015-3024.

48 D. P. Rillema, J. K. Nagle, L. F. Barringer Jr and T. J. Meyer, J. Am. Chem. Soc., 1981, 103, 56-62.

49 K. M. Kadish, D. J. Leggett and D. Chang, Inorg. Chem., 1982, 21, 3618-3622. 
50 K. M. Kadish and D. Chang, Inorg. Chem., 1982, 21, 3614-3618. 51 S. Chattopadhyay, C. V. Kumar and P. Das, Chem. Phys. Lett., 1983, 98, 250-254.

52 V. Gray, D. Dzebo, A. Lundin, J. Alborzpour, M. Abrahamsson, B. Albinsson and K. Moth-Poulsen, J. Mater. Chem. C, 2015, 3, 11111-11121.

53 J. Andraos, J. Chem. Educ., 1999, 76, 1578-1583.

54 L. M. A. Levine and D. Holten, J. Phys. Chem., 1988, 92, 714-720.

55 J. Vura-Weis, S. H. Abdelwahed, R. Shukla, R. Rathore, M. A. Ratner and M. R. Wasielewski, Science, 2010, 328, 1547-1550.

56 N. I. Nijegorodov, W. S. Downey and M. B. Danailov, Spectrochim. Acta, Part A, 2000, 56, 783-795.
57 E. A. Weiss, M. J. Ahrens, L. E. Sinks, A. V. Gusev, M. A. Ratner and M. R. Wasielewski, J. Am. Chem. Soc., 2004, 126, 5577-5584.

58 E. A. Weiss, M. J. Tauber, R. F. Kelley, M. J. Ahrens, M. A. Ratner and M. R. Wasielewski, J. Am. Chem. Soc., 2005, 127, 11842-11850.

59 T. Mani, D. M. Niedzwiedzki and S. A. Vinogradov, J. Phys. Chem. A, 2012, 116, 3598-3610.

60 K. Kilså, J. Kajanus, J. Mårtensson and B. Albinsson, J. Phys. Chem. B, 1999, 103, 7329-7339.

61 K. Pettersson, A. Kyrychenko, E. Ro and T. Ljungdahl, J. Phys. Chem. A, 2006, 110, 310-318.

62 D. Dzebo, K. Börjesson, V. Gray, K. Moth-Poulsen and B. Albinsson, J. Phys. Chem. C, 2016, 120, 23397-23406. 